\title{
Eight-and-a-Half Syndrome as The First Presentation of Unknown Metastatic Primary Tumor
}

\author{
Costa e Silva $\mathbf{M}^{1 *}$, Mota $\mathrm{C}^{2}$, Silva $\mathrm{E}^{1,3}$ and Barroso $\mathrm{A}^{1,3}$ \\ ${ }^{1}$ Department of Pulmonology, Centro Hospitalar Vila Nova de Gaia/Espinho, Portugal \\ ${ }^{2}$ Department of Neurology, Centro Hospitalar Vila Nova de Gaia/Espinho, Portugal \\ ${ }^{3}$ Thoracic Tumors Multidisciplinary Unit, Pulmonology Department, Vila Nova de Gaia-Espinho Hospital Center \\ *Corresponding author: Margarida Costa e Silva; Rua Agro de Moinhos 168 D4.1, 4430-004 Mafamude, Portugal \\ To Cite This Article: Costa e Silva M, Mota C, Silva E, Barroso A. Eight-and-a-Half Syndrome as The First Presentation of Unknown Metastatic \\ Primary Tumor. Am J Biomed Sci \& Res. 2021 - 13(6). AJBSR.MS.ID.001925. DOI: 10.34297/AJBSR.2021.13.001925.
}

Received: 眥 July 26, 2021; Published: 觜 August 12, 2021

\begin{abstract}
The eight-and-a-half syndrome (EHS) is a rare brainstem syndrome. We present a case of a 48-year-old man with a 5-day history of visual disturbance, headache and dizziness of sudden onset concomitantly with a unique presentation of a conjugate horizontal gaze palsy, an ipsilateral internuclear ophthalmoplegia and an ipsilateral lower motor neuron-like facial palsy. Magnetic resonance imaging revealed several lesions of nodular morphology, with hypersignal in T2 and FLAIR, with signal reinforcement after gadolinium administration (complete ring morphology). A Thoracic CT revealed a lesion with spiculate contours on the right lower lobe together with a right perihilar and mediastinal infiltrative lesion and a large pre-tracheal, infracarinal adenopathic conglomerate. An aspiration biopsy of left axillary nodes allowed the diagnosis of small-cell lung cancer (SCLC), staged as cT4N3M1c-IVB. Treatment with corticosteroid therapy as well as holocranial radiotherapy for the brain metastases and chemotherapy was started, with progressive improvement and total resolution of EHS after one month of treatment. This is the first case describing an EHS as the initial clinical presentation of brain metastases. The authors would like to highlight the importance of recognizing the EHS as the first presentation of a serious non-neurological primary disease that needs urgent multidisciplinary guidance.
\end{abstract}

Keywords: Eight-and-a-half syndrome, Brain metastases, Lung cancer

Abbreviations: CT: Computerized Tomography; ECG: Electrocardiogram; ED: Emergency Department; EHS: Eight-and-a-Half-Syndrome; FLAIR: Fluid Attenuated Inversion Recovery; HBV: Hepatitis B Virus; HCV: Hepatitis C Virus; HIV: Human Immunodeficiency Virus; MRI: Magnetic Resonance Imaging; SCLC: Small-Cell Lung Cancer

\section{Introduction}

Eight-and-a-half syndrome (EHS) is a rare pontine neuroophthalmic syndrome consisting of conjugate horizontal gaze palsy, ipsilateral internuclear ophthalmoplegia and ipsilateral peripheral facial nerve palsy $[1,2]$. The most common etiology is ischemic stroke; less common etiologies are demyelinating lesions and space occupying lesions [3]. Lung cancer is the most common cause of cancer-related mortality in humans, being responsible for more or less $50 \%$ of all brain metastases [4].

\section{Case Description}

The authors report a case of a 48-year-old Caucasian male with smoking history, presenting to the emergency department (ED) with a 5-day history of visual disturbance, headache and dizziness of sudden onset. Past medical history was significant for ventricular extra systoles and mitral prolapse (medicated with bisoprolol $5 \mathrm{mg}$ id). The patient had no previous history of neurological disorders. Affirmed tinnitus (mainly on the left) and denied fever, nausea, vomit or others.

Neurological examination at admission to ED revealed left horizontal conjugate gaze palsy, limited adduction of the left eye, and horizontal nystagmus of the right eye upon abduction. Vertical eye movements were normal. Pupils were symmetrical and reactive to light. Left peripheral facial palsy was observed. No hemiparesis or hemisensory deficit was found, and deep tendon reflexes were 
normal. No pathological Babinski's sign was induced and no sign of meningeal irritation was observed. Left axillary lymphadenopathy was found at physical examination.

Lab analysis showed no remarks and ECG presented sinus rhythm with heart rate of $75 \mathrm{bpm}$. Brain CT revealed subcortical hypodensity in the left cerebellar hemisphere with rounded morphology and without significant mass effect. Cerebral CT angiography was performed, showing no major changes.

After admission for further investigation, autoimmune screen and serologies for HIV, HBV and HCV were found to be negative. A brain magnetic resonance imaging (MRI) was performed showing several lesions of nodular morphology, with hypersignal in T2 and FLAIR, with signal reinforcement after gadolinium administration (complete ring morphology) (Figure 1A). These lesions involved the posterior slope of the protuberance reaching the left facial nerve colliculi, the ipsilateral cerebellar hemisphere parenchyma, the white substance adjacent to the frontal horn of the left lateral ventricle, the white substance of the posterior third of the right semi-oval center and the cortex parasagittal frontal view on the same side (Figure 1B \& 1C).
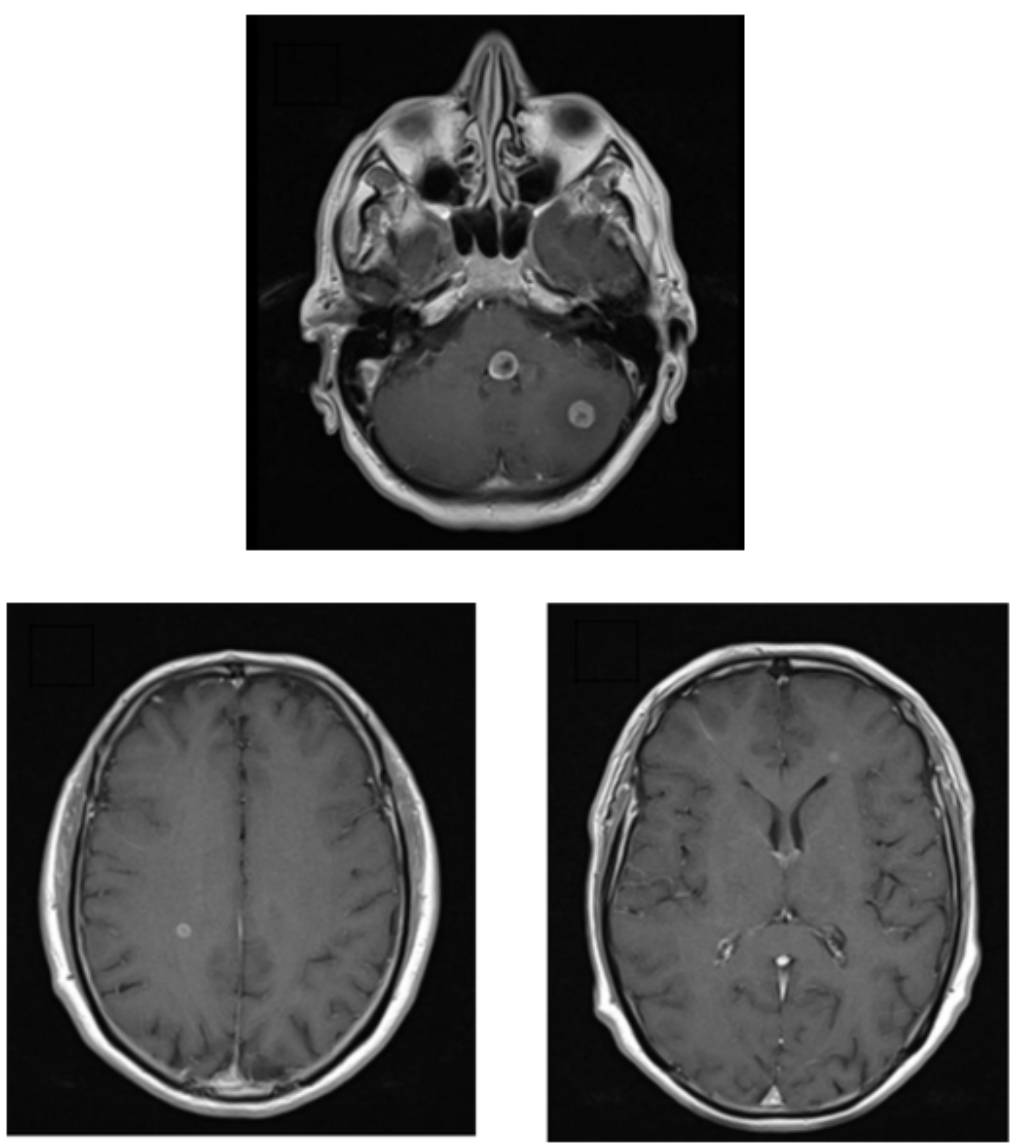

Figure 1: Brain MRI - Coronal T1 with gadolinium: A - Lesions with nodular morphology involving the left facial nerve colliculi and the ipsilateral cerebellar hemisphere parenchyma; B - Lesion involving the white substance of the posterior third of the right semi-oval center; $C$ - Lesion involving the white substance adjacent to the frontal horn of the left lateral ventricle.

MRI findings were suggestive of secondary lesions and a thoracic CT was performed, revealing a hypocaptive lesion with spiculate contours in the apical segment of right lower lobe with sub pleural location, presenting suspicious characteristics; concomitantly, a right perihilar and mediastinal infiltrative lesion and a large pre-tracheal, infracarinal adenopathic conglomerate, with extension to the right hilum, were identified, accompanied by enlargement of the left axillary nodes.

Aspiration biopsy of left axillary nodes allowed the diagnosis of small-cell lung cancer (SCLC), staged as cT4N3M1c-IVB. The patient was started on prednisolone $25 \mathrm{mg}$ id and holocranial radiotherapy for the brain metastases later on; difficulties in weaning off corticosteroid therapy were observed. First-line chemotherapy with carboplatin plus etoposide was started but, due to disease progression, the patient has taken so far four lines of chemotherapy and awaits permission for lurbinectedin administration. After treatment initiation, the patient immediately began to improve with total resolution of EHS occurring after one month of treatment. 


\section{Discussion}

EHS is a rare brainstem syndrome which localizes to the caudal tegmental region of the pons, and is defined by the combination of a seventh cranial nerve palsy and an ipsilateral one-and-a-half syndrome [3,5]. To best of our knowledge, this is the first case describing an EHS as the initial clinical presentation of brain metastases of any organ.

A recent review of the literature for published cases of EHS found a total of 27 cases, with cerebrovascular disease being most frequent underlying etiology (17 cases), followed by demyelinating disease ( 9 cases); only one case described an infectious disease as underlying etiology, a tuberculoma [3].

EHS caused by space-occupying lesion at the level of the pons, such as in our case, is an even rarer event described only in a tuberculoma case [2,6]. Early recognition of syndrome's features, allows a proper and quick diagnosis avoiding treatment delays [2]. The treatment is directly correlated with the underlying condition as well as neurorehabilitation [1,2]. Commonly, facial palsy is the first to improve followed by the ocular symptoms [1]. Recovery tends to be incomplete for those with a vascular-related EHS and almost complete for those of non-vascular etiology [3]. Time to onset of symptoms might suggest the cause, as for instance sudden onset can favor ischemic stroke [1,2]. Current literature emphasizes the importance of etiological assessment [3]. In our report neither the symptoms nor time to onset could suggest the etiology and the syndrome was proved to be caused by a rare event, a tumor located at the pons level (a space-occupying lesion).

\section{Conclusion}

We intend to highlight the importance of recognizing the Eightand-a-half syndrome as the first presentation of a serious nonneurological primary disease that needs urgent multidisciplinary guidance.

\section{References}

1. Ahmed HA, Al Assaf OY, Alzarooni HM, Sarathchandran P (2020) Clinical findings of eight-and-a-half syndrome, Oxford Medical Case Reports 2020(10): 1-4.

2. Mesina BVQ, Sosuan GMN, Reyes KB (2018) Eight-and-a-half syndrome: a rare potentially life-threatening disease. GMS Ophthalmol Cases 8: Doc04..

3. Cárdenas-Rodríguez MA, Castillo-Torres SA (2020) Eight-and-a-half syndrome: video evidence and updated literature review. BMJ Case Rep 13(5): e234075.

4. Yousefi M, Bahrami T, Salmaninejad A, Nosrati R, Ghaffari P, et al. (2017) Lung cancer-associated brain metastasis: Molecular mechanisms and therapeutic options. Cell Oncol (Dordr) 40(5): 419-441.

5. Xia NG, Chen YY, Li J, Chen X, Ye ZS, et al. (2018) Eight-and-a-half syndrome caused by a pontine haemorrhage: a case report and review of the literature. Int J Neurosci 128(8): 746-750.

6. van Toorn R, Schoeman JF, Donald PR (2006) Brainstem tuberculoma presenting as eight-and-a-half syndrome. Eur J Paediatr Neurol 10(1): 41-44. 